\title{
A NEW CLASS OF INTEGRALS INVOLVING EXTENDED MITTAG-LEFFLER FUNCTIONS
}

\author{
G. RAHMAN, A. GHAFFAR, K.S. NISAR* AND S. MUBEEN
}

\begin{abstract}
The main aim of this paper is to establish two generalized integral formulas involving the extended Mittag-Leffler function based on the well known Lavoie and Trottier integral formula and the obtain results are express in term of extended Wright-type function. Also, we establish certain special cases of our main result.
\end{abstract}

\section{INTRODUCTION}

The Mittag-Leffler functions $E_{\alpha}(z)$ and $E_{\alpha, \beta}(z)$ are defined by the following series:

$$
E_{\alpha}(z)=\sum_{n=0}^{\infty} \frac{z^{n}}{\Gamma(\alpha n+1)}, z \in \mathbb{C}, \Re(\alpha)>0,
$$

and

$$
E_{\alpha, \beta}(z)=\sum_{n=0}^{\infty} \frac{z^{n}}{\Gamma(\alpha n+\beta)}, z, \beta \in \mathbb{C}, \Re(\alpha)>0,
$$

respectively. For the generalizations and applications of Mittag-Leffler function, the readers can refer to the recent work of researchers $([4,5,6,7,8,10,20,22,30])$, Kilbas et al. ([11], Chapter 1) and Saigo and Kilbas [12]. In recent years, the Mittag-Leffler function and some of its variety generalizations have been numerically established in the complex plane $([9,24])$. A new generalization of the Mittag-Leffler functions $E_{\alpha, \beta}(z)$ of $(1.2)$ have defined by Prabhakar [21] as follows:

$$
E_{\alpha, \beta}^{\gamma}(z)=\sum_{n=0}^{\infty} \frac{(\gamma)_{n}}{\Gamma(\alpha n+\beta)} \frac{z^{n}}{n !}, z, \beta \in \mathbb{C}, \Re(\alpha)>0,
$$

where $(\gamma)_{n}$ denote the well known Pochhammer Symbol which is defined by:

$$
(\gamma)_{n}=\left\{\begin{array}{l}
1, \quad(n=0, \gamma \in \mathbb{C}) \\
\gamma(\gamma+1)(\gamma+2) \ldots(\gamma+n-1)(n \in N, \gamma \in \mathbb{C})
\end{array}\right.
$$

2010 Mathematics Subject Classification. 33B20, 33C20, 33C45, 33C60, 33B15, 33C05.

Key words and phrases. extended Mittag-Leffler function; Wright-type hypergeometric functions; extended Wright-type hypergeometric functions.

* Corresponding author. 
In fact, the following special cases are satisfied:

$$
E_{\alpha, \beta}^{1}(z)=E_{\alpha, \beta}(z), E_{\alpha, 1}^{1}(z)=E_{\alpha, \beta}(z) .
$$

Recently, many researchers have established the significance and great consideration of Mittag-Leffler functions in the theory of special functions for exploring the generalizations and some applications. Various extensions for these functions are found in ([8], [25, 26, 27]). Srivastava and Tomovski [29] have defined further generalization of the Mittag-Leffler function $E_{\alpha, \beta}^{1}(z)$ of (3), which is defined as:

$$
E_{\alpha, \beta}^{\gamma, k}(z)=\sum_{n=0}^{\infty} \frac{(\gamma)_{n k}}{\Gamma(\alpha n+\beta)} \frac{z^{n}}{n !},
$$

where $z, \alpha, \beta \in \mathbb{C} ; \Re(\alpha)>\max \{0, \Re(k)-1\} ; \Re(k)>0$. Very recently, Ozarslan and Yilmaz [19] have investigated an extended Mittag-Leffler function $E_{\alpha, \beta}^{\gamma, c}(z: p)$ which is defined as:

$$
E_{\alpha, \beta}^{\gamma ; c}(z)=\sum_{n=0}^{\infty} \frac{\mathbf{B}_{p}(\gamma+n, c-\gamma)(c)_{n}}{\mathbf{B}(\gamma, c-\gamma) \Gamma(\alpha n+\beta)} \frac{z^{n}}{n !},
$$

where $p>0, \Re(c)>\Re(\gamma)>0$ and $B_{p}(x, y)$ is extended beta function defined in Mainardi as follows:

$$
B_{p}(x, y)=\int_{0}^{t} t^{(x-1)}(1-t)^{(y-1)} e^{-(p / t(1-t))} d t
$$

where $\Re(p)>0, \Re(x)>0$ and $\Re(y)>0$. If $p=0$, then $B_{p}(x, y)$ reduces to the following beta function:

$$
B(x, y)=\int_{0}^{t} t^{(x-1)}(1-t)^{(y-1)} d t
$$

The gamma function is defined by:

$$
\Gamma(z)=\int_{0}^{t} t^{(z-1)} e^{(-t)} d t ; \quad \Re(z)>0 .
$$

By inspection, we conclude the following relation

$$
\Gamma(z+1)=z \Gamma(z) .
$$

The generalized hypergeometric function ${ }_{p} F_{q}(z)$ is defined in [6] as:

$$
\begin{aligned}
{ }_{p} F_{q}(z) & ={ }_{p} F_{q}\left[\begin{array}{c}
\left(a_{1}\right),\left(a_{2}\right), \ldots,\left(a_{p}\right) ; \\
\left(b_{1}\right),\left(b_{2}\right), \ldots,\left(b_{q}\right) ;
\end{array}\right] \\
& =\sum_{n=0}^{\infty} \frac{\left(a_{1}\right)_{n}\left(a_{2}\right)_{n} \ldots\left(a_{q}\right)_{n} z^{n}}{\left(b_{1}\right)_{n}\left(b_{2}\right)_{n} \ldots\left(b_{p}\right)_{n} n !}
\end{aligned}
$$


A NEW CLASS OF INTEGRALS INVOLVING EXTENDED MITTAG-LEFFLER FUNCTIONS where $\alpha_{i}, \beta_{i} \in \mathbb{C} ; i=1,2, \ldots, p ; j=1,2, \ldots, q$ and $b_{j} \neq 0,-1,-2, \ldots$ and $(z)_{n}$ is the Pochhammer symbols.

Sharma and Devi [23] defined extended Wright type function in the following series:

$$
\begin{aligned}
{ }_{p+1} F_{q+1}(z ; p) & ={ }_{p+1} F_{q+1}\left[\begin{array}{c}
\left(a_{i}, A_{i}\right)_{1, p},(\gamma, 1) ; \\
\left(b_{j}, \beta_{j}\right)_{1, p},(c, 1) ;
\end{array}\right] \\
& =\sum_{n=0}^{\infty} \frac{\Gamma\left(a_{1}+A_{1} n\right) \ldots \Gamma\left(a_{p}+A_{p} n\right) \mathbf{B}_{p}(\gamma+n, c-\gamma) z^{n}}{\Gamma\left(b_{1}+\beta_{1} n\right) \ldots \Gamma\left(b_{q}+\beta_{q} n\right) \Gamma(c-\gamma) n !}
\end{aligned}
$$

where $i=1,2, \ldots, p, j=1,2, \ldots, q, \Re(p)>0, \Re(c)>\Re(\gamma)>0$ and $p \geq 0$. Recently, Mittal et al. [15] defined an extended generalized Mittag-Leffer function as:

$$
E_{\alpha, \beta}^{\gamma, q ; c}(z ; p)=\sum_{n=0}^{\infty} \frac{\mathbf{B}_{p}(\gamma+n q, c-\gamma)(c)_{n q}}{\mathbf{B}(\gamma, c-\gamma) \Gamma(\alpha n+\beta)} \frac{z^{n}}{n !}
$$

where $\alpha, \beta, \gamma \in \mathbb{C}, \Re(\alpha)>0, \Re(\beta)>0, \Re(\gamma)>0, q>0$ and $B_{p}(x, y)$ is an extended beta function defined in (1.7). In this paper, we derive new integral formulas involving the Mittag-Leffler function (1.6) and (1.13). For the present investigation, we need the following result of Lavoie and Trottier [13]

$$
\int_{0}^{1} x^{(a-1)}(1-x)^{(2 b-1)}\left(1-\frac{x}{3}\right)^{(2 a-1)}\left(1-\frac{x}{4}\right)^{(b-1)} d x=\left(\frac{2}{3}\right)^{2 a} \frac{\Gamma(a) \Gamma(b)}{\Gamma(a+b)},
$$

where $\Re(a)>0, \Re(b)>0$.

For more details about the recent works in the field of dynamical systems theory, stochastic systems, non-equilibrium statistical mechanics and quantum mechanics, the readers may refer to the recent work of the researchers $[1,2,3,14,16,17,18]$ and the references cited therein.

\section{Main Result}

In this section, the generalized integral formulas involving the Mittag-Leffler function (1.6) and (1.13), are established here by inserting with the suitable argument in the integrand of (1.14).

Theorem 1. Let $y, c, \rho, j, \alpha, \beta, \gamma \in \mathbb{C}$; with $\Re(\alpha)>\max \{0, \Re(c)-1\} ; \Re(c)>0 . \Re(\gamma)>$ $0, \Re(2 \rho+j)>0$ and $x>0$. Then the following formula holds true:

$$
\int_{0}^{1} x^{(\rho+j-1)}(1-x)^{(2 \rho-1)}\left(1-\frac{x}{3}\right)^{(2(\rho+j)-1)}\left(1-\frac{x}{4}\right)^{(\rho-1)} E_{\alpha, \beta}^{\gamma ; c}\left(y\left(1-\frac{x}{4}\right)(1-x)^{2}\right) d x
$$




$$
=\left(\frac{2}{3}\right)^{2(\rho+j)} \frac{\Gamma(\rho+j)}{\Gamma(\gamma)}{ }_{3} \psi_{3}\left[\begin{array}{ll}
(\rho, 1),(c, 1),(\gamma, 1) ; & \mid y ; p \\
(\beta, \alpha),(2 \rho+j, 1),(c, 1) ; &
\end{array}\right]
$$

Proof. Let $S_{1}$ be the left-hand side of (2.1). Now applying the series representation of the Mittag-Leffler function (1.6) to the integrand of (1.14) and by interchanging the order of integration and summation under the given condition in Theorem (1), we have

$$
\begin{aligned}
S_{1}= & \int_{0}^{1} x^{(\rho+j-1)}(1-x)^{(2 \rho-1)}\left(1-\frac{x}{3}\right)^{(2(\rho+j)-1)}\left(1-\frac{x}{4}\right)^{(\rho-1)} E_{\alpha, \beta}^{\gamma ; c}\left(y\left(1-\frac{x}{4}\right)(1-x)^{2}\right) d x \\
= & \int_{0}^{1} x^{(\rho+j-1)}(1-x)^{(2 \rho-1)}\left(1-\frac{x}{3}\right)^{(2(\rho+j)-1)}\left(1-\frac{x}{4}\right)^{(\rho-1)} \\
& \times \sum_{n=0}^{\infty} \frac{\mathbf{B}_{p}(\gamma+n, c-\gamma)(c)_{n}}{\mathbf{B}(\gamma, c-\gamma) \Gamma(\alpha n+\beta)} \frac{\left(y\left(1-\frac{x}{4}\right)(1-x)^{2}\right)^{n}}{n !} d x \\
= & \int_{0}^{1} x^{(\rho+j-1)}(1-x)^{2(\rho+n)-1}\left(1-\frac{x}{3}\right)^{(2(\rho+j)-1)}\left(1-\frac{x}{4}\right)^{(\rho+n-1)} \\
& \times \sum_{n=0}^{\infty} \frac{\mathbf{B}_{p}(\gamma+n, c-\gamma)(c)_{n}}{\mathbf{B}(\gamma, c-\gamma) \Gamma(\alpha n+\beta)} \frac{y^{n}}{n !} d x .
\end{aligned}
$$

Now using (1.6) and (1.14), we get

$$
\begin{aligned}
S_{1} & =\left(\frac{2}{3}\right)^{2(\rho+j)} \frac{\Gamma(\rho+j) \Gamma(\rho+n)}{\Gamma(2 \rho+j+n)} \sum_{n=0}^{\infty} \frac{\mathbf{B}_{p}(\gamma+n, c-\gamma)(c)_{n}}{\mathbf{B}(\gamma, c-\gamma) \Gamma(\alpha n+\beta)} \frac{y^{n}}{n !} \\
& =\left(\frac{2}{3}\right)^{(2 \rho+j)} \frac{\Gamma(\rho+j)}{\Gamma(\gamma)} \sum_{n=0}^{\infty} \frac{\mathbf{B}_{p}(\gamma+n, c-\gamma) \Gamma(\rho+n) \Gamma(c+n)}{\Gamma(c-\gamma) \Gamma(\alpha n+\beta) \Gamma(2 \rho+j+n)} \frac{y^{n}}{n !}
\end{aligned}
$$

which upon using (1.12), we get

$$
S_{1}=\left(\frac{2}{3}\right)^{2(\rho+j)} \frac{\Gamma(\rho+j)}{\Gamma(\gamma)}{ }_{3} \psi_{3}\left[\begin{array}{ll}
(\rho, 1),(c, 1),(\gamma, 1) & ; \mid y ; p \\
(\beta, \alpha),(2 \rho+j, 1),(c, 1) &
\end{array}\right] .
$$

Theorem 2. Let $y, c, \rho, j, \alpha, \beta, \gamma \in \mathbb{C}$; with $\Re(\alpha)>\max \{0, \Re(c)-1\} ; \Re(c)>0$. $\Re(\gamma)>$ $0, \Re(2 \rho+j)>0$ and $x>0$. Then the following formula holds true:

$$
\begin{gathered}
\int_{0}^{1} x^{(\rho-1)}(1-x)^{2(\rho+j)-1}\left(1-\frac{x}{3}\right)^{(2 \rho-1)}\left(1-\frac{x}{4}\right)^{(\rho+j-1)} E_{\alpha, \beta}^{\gamma ; c}\left(y x\left(1-\frac{x}{3}\right)^{2} ; p\right) d x \\
=\left(\frac{2}{3}\right)^{2(\rho)} \frac{\Gamma(\rho+j)}{\Gamma(\gamma)}{ }_{3} \psi_{3}\left[\begin{array}{c}
(\rho, 1),(c, 1),(\gamma, 1) ; \\
(\beta, \alpha),(2 \rho+j, 1),(c, 1) ;
\end{array} ; \mid \frac{4}{9} y ; p\right] .
\end{gathered}
$$


A NEW CLASS OF INTEGRALS INVOLVING EXTENDED MITTAG-LEFFLER FUNCTIONS

Proof. Let $S_{2}$ be the left-hand side of (2.2). Now applying the series representation of the Mittag-Leffler function (1.6) to the integrand of (1.14), we have

$$
\begin{aligned}
S_{2}= & \int_{0}^{1} x^{(\rho-1)}(1-x)^{2(\rho+j)-1}\left(1-\frac{x}{3}\right)^{(2 \rho-1)}\left(1-\frac{x}{4}\right)^{(\rho+j-1)} E_{\alpha, \beta}^{\gamma ; c}\left(y x\left(1-\frac{x}{3}\right)^{2} ; p\right) d x \\
= & \int_{0}^{1} x^{(\rho-1)}(1-x)^{2(\rho+j)-1}\left(1-\frac{x}{3}\right)^{(2 \rho-1)}\left(1-\frac{x}{4}\right)^{(\rho+j-1)} \\
& \times \sum_{n=0}^{\infty} \frac{\mathbf{B}_{p}(\gamma+n, c-\gamma)(c)_{n}}{\mathbf{B}(\gamma, c-\gamma) \Gamma(\alpha n+\beta)} \frac{\left(y\left(1-\frac{x}{3}\right)^{2}\right)^{n}}{n !} d x \\
= & \int_{0}^{1} x^{(\rho+n-1)}(1-x)^{2(\rho+j)-1}\left(1-\frac{x}{3}\right)^{2(\rho+n)-1}\left(1-\frac{x}{4}\right)^{(\rho+j-1)} \\
& \times \sum_{n=0}^{\infty} \frac{\mathbf{B}_{p}(\gamma+n, c-\gamma)(c)_{n}}{\mathbf{B}(\gamma, c-\gamma) \Gamma(\alpha n+\beta)} \frac{y^{n}}{n !} d x
\end{aligned}
$$

Now using (1.6) and (1.14), we get

$$
\begin{aligned}
S_{2} & =\left(\frac{2}{3}\right)^{2(\rho+n)} \frac{\Gamma(\rho+j) \Gamma(\rho+n)}{\Gamma(2 \rho+j+n)} \sum_{n=0}^{\infty} \frac{\mathbf{B}_{p}(\gamma+n, c-\gamma)(c)_{n}}{\mathbf{B}(\gamma, c-\gamma) \Gamma(\alpha n+\beta)} \frac{y^{n}}{n !} \\
& =\left(\frac{2}{3}\right)^{2 \rho} \frac{\Gamma(\rho+j)}{\Gamma(\gamma)} \sum_{n=0}^{\infty} \frac{\left.\mathbf{B}_{p}(\gamma+n, c-\gamma) \Gamma(\rho+n) \Gamma(c+n)\right)}{\Gamma(c-\gamma) \Gamma(\alpha n+\beta) \Gamma(2 \rho+j+n)} \frac{\left(\frac{4}{9} y\right)^{n}}{n !},
\end{aligned}
$$

which upon using (1.12), we get

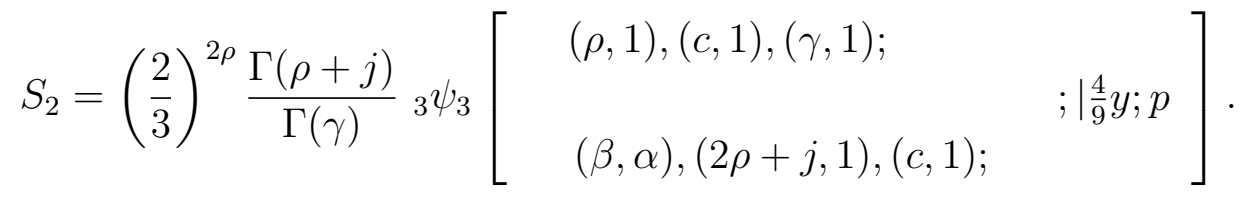

Theorem 3. Let $y, c, \rho, j, \alpha, \beta, \gamma \in \mathbb{C}$; with $\Re(\alpha)>\max \{0, \Re(c)-1\} ; \Re(c)>0$. $\Re(\gamma)>$ $0, \Re(2 \rho+j)>0$ and $x>0$. Then the following formula holds true:

$$
\begin{gathered}
\int_{0}^{1} x^{(\rho+j-1)}(1-x)^{(2 \rho-1)}\left(1-\frac{x}{3}\right)^{(2(\rho+j)-1)}\left(1-\frac{x}{4}\right)^{(\rho-1)} E_{\alpha, \beta}^{\gamma, q ; c}\left(y\left(1-\frac{x}{4}\right)(1-x)^{2} ; p\right) d x \\
=\left(\frac{2}{3}\right)^{2(\rho+j)} \frac{\Gamma(\rho+j)}{\Gamma(\gamma)}{ }_{3} \psi_{3}\left[\begin{array}{c}
(\rho, 1),(c, q),(\gamma, 1) ; \\
(\beta, \alpha),(2 \rho+j, 1),(c, 1) ;
\end{array} ; \mid y ; p\right]
\end{gathered}
$$

Proof. Let $S_{3}$ be the left-hand side of (2.3). Now applying the series representation of the Mittag-Leffler function (1.6) to the integrand of (1.14) and by interchanging the order of 
integration and summation under the given condition in Theorem (1), we have

$$
\begin{aligned}
S_{3}= & \int_{0}^{1} x^{(\rho+j-1)}(1-x)^{(2 \rho-1)}\left(1-\frac{x}{3}\right)^{(2(\rho+j)-1)}\left(1-\frac{x}{4}\right)^{(\rho-1)} E_{\alpha, \beta}^{\gamma, q ; c}\left(y\left(1-\frac{x}{4}\right)(1-x)^{2} ; p\right) d x \\
= & \int_{0}^{1} x^{(\rho+j-1)}(1-x)^{(2 \rho-1)}\left(1-\frac{x}{3}\right)^{(2(\rho+j)-1)}\left(1-\frac{x}{4}\right)^{(\rho-1)} \\
& \times \sum_{n=0}^{\infty} \frac{\mathbf{B}_{p}(\gamma+n q, c-\gamma)(c)_{n q}}{\mathbf{B}(\gamma, c-\gamma) \Gamma(\alpha n+\beta)} \frac{\left(y\left(1-\frac{x}{4}\right)(1-x)^{2}\right)^{n}}{n !} d x \\
= & \int_{0}^{1} x^{(\rho+j-1)}(1-x)^{2(\rho+n)-1}\left(1-\frac{x}{3}\right)^{(2(\rho+j)-1)}\left(1-\frac{x}{4}\right)^{(\rho+n-1)} \\
& \times \sum_{n=0}^{\infty} \frac{\mathbf{B}_{p}(\gamma+n q, c-\gamma)(c)_{n q}}{\mathbf{B}(\gamma, c-\gamma) \Gamma(\alpha n+\beta)} \frac{y^{n}}{n !} d x .
\end{aligned}
$$

Now using (1.6) and (1.14), we get

$$
\begin{aligned}
S_{1} & =\left(\frac{2}{3}\right)^{2(\rho+j)} \frac{\Gamma(\rho+j) \Gamma(\rho+n)}{\Gamma(2 \rho+j+n)} \sum_{n=0}^{\infty} \frac{\mathbf{B}_{p}(\gamma+n q, c-\gamma)(c)_{n q}}{\mathbf{B}(\gamma, c-\gamma) \Gamma(\alpha n+\beta)} \frac{y^{n}}{n !} \\
& =\left(\frac{2}{3}\right)^{(2 \rho+j)} \frac{\Gamma(\rho+j)}{\Gamma(\gamma)} \sum_{n=0}^{\infty} \frac{\mathbf{B}_{p}(\gamma+n q, c-\gamma) \Gamma(\rho+n) \Gamma(c+n q)}{\Gamma(c-\gamma) \Gamma(\alpha n+\beta) \Gamma(2 \rho+j+n)} \frac{y^{n}}{n !},
\end{aligned}
$$

which upon using (1.12), we get

$$
S_{3}=\left(\frac{2}{3}\right)^{2(\rho+j)} \frac{\Gamma(\rho+j)}{\Gamma(\gamma)}{ }_{3} \psi_{3}\left[\begin{array}{ll}
(\rho, 1),(c, 1),(\gamma, 1) ; & ; \mid y ; p \\
(\beta, \alpha),(2 \rho+j, 1),(c, 1) ; &
\end{array}\right]
$$

Theorem 4. Let $\rho, j, \alpha, \beta, \gamma \in \mathbb{C}$; with $R(\alpha)>0, \Re(c)>\Re(\gamma)>0, \Re(2 \rho+j)>0$ and $x>0$. Then the following formula holds true:

$$
\begin{gathered}
\int_{0}^{1} x^{(\rho-1)}(1-x)^{2(\rho+j)-1}\left(1-\frac{x}{3}\right)^{(2 \rho-1)}\left(1-\frac{x}{4}\right)^{(\rho+j-1)} E_{\alpha, \beta}^{\gamma, q ; c}\left(y x\left(1-\frac{x}{3}\right)^{2} ; p\right) d x \\
=\left(\frac{2}{3}\right)^{2(\rho)} \frac{\Gamma(\rho+j)}{\Gamma(\rho)}{ }_{3} \psi_{3}\left[\begin{array}{c}
(\rho, 1),(c, q),(\gamma, 1) ; \\
(\beta, \alpha),(2 \rho+j, 1),(c, 1) ;
\end{array} ; \mid \frac{4}{9} y ; p\right] .
\end{gathered}
$$

Proof. Let $S_{4}$ be the left-hand side of (2.4). Now applying the series representation of the Mittag-Leffler function (1.6) to the integrand of (1.14), we have

$$
S_{4}=\int_{0}^{1} x^{(\rho-1)}(1-x)^{2(\rho+j)-1}\left(1-\frac{x}{3}\right)^{(2 \rho-1)}\left(1-\frac{x}{4}\right)^{(\rho+j-1)} E_{\alpha, \beta}^{\gamma, q ; c}\left(y x\left(1-\frac{x}{3}\right)^{2} ; p\right) d x
$$


A NEW CLASS OF INTEGRALS INVOLVING EXTENDED MITTAG-LEFFLER FUNCTIONS

$$
\begin{aligned}
= & \int_{0}^{1} x^{(\rho-1)}(1-x)^{2(\rho+j)-1}\left(1-\frac{x}{3}\right)^{(2 \rho-1)}\left(1-\frac{x}{4}\right)^{(\rho+j-1)} \\
& \times \sum_{n=0}^{\infty} \frac{\mathbf{B}_{p}(\gamma+n q, c-\gamma)(c)_{n q}}{\mathbf{B}(\gamma, c-\gamma) \Gamma(\alpha n+\beta)} \frac{\left(y\left(1-\frac{x}{3}\right)^{2}\right)^{n}}{n !} d x \\
= & \int_{0}^{1} x^{(\rho+n-1)}(1-x)^{2(\rho+j)-1}\left(1-\frac{x}{3}\right)^{2(\rho+n)-1}\left(1-\frac{x}{4}\right)^{(\rho+j-1)} \\
& \times \sum_{n=0}^{\infty} \frac{\mathbf{B}_{p}(\gamma+n q, c-\gamma)(c)_{n}}{\mathbf{B}(\gamma, c-\gamma) \Gamma(\alpha n+\beta)} \frac{y^{n}}{n !} d x
\end{aligned}
$$

Now using (1.13) and (1.14), we get

$$
\begin{aligned}
S_{4} & =\left(\frac{2}{3}\right)^{2(\rho+n)} \frac{\Gamma(\rho+j) \Gamma(\rho+n)}{\Gamma(2 \rho+j+n)} \sum_{n=0}^{\infty} \frac{\mathbf{B}_{p}(\gamma+n q, c-\gamma)(c)_{n q}}{\mathbf{B}(\gamma, c-\gamma) \Gamma(\alpha n+\beta)} \frac{y^{n}}{n !} \\
& =\left(\frac{2}{3}\right)^{2 \rho} \frac{\Gamma(\rho+j)}{\Gamma(\gamma)} \sum_{n=0}^{\infty} \frac{\mathbf{B}_{p}(\gamma+n q, c-\gamma) \Gamma(\rho+n) \Gamma(c+n q)}{\Gamma(c-\gamma) \Gamma(\alpha n+\beta) \Gamma(2 \rho+j+n)} \frac{\left(\frac{4}{9} y\right)^{n}}{n !}
\end{aligned}
$$

which upon using (1.12), we get

$$
S_{4}=\left(\frac{2}{3}\right)^{2 \rho} \frac{\Gamma(\rho+j)}{\Gamma(\gamma)}{ }_{3} \psi_{3}\left[\begin{array}{ll}
(\rho, 1),(c, q),(\gamma, 1) ; & \mid \frac{4}{9} y ; p \\
(\beta, \alpha),(2 \rho+j, 1),(c, 1) ; &
\end{array}\right]
$$

\section{Particular cases}

In this section, we obtained some particular cases of Theorems 1-4. Setting $p=0$ in (1.6) and (1.13), then we get the following well known Mittag-Leffler functions.

$$
E_{\alpha, \beta}^{\gamma, c}(z)=\sum_{n=0}^{\infty} \frac{\mathbf{B}_{p}(\gamma+n, c-\gamma)(c)_{n}}{\mathbf{B}(\gamma, c-\gamma) \Gamma(\alpha n+\beta)} \frac{z^{n}}{n !}
$$

and

$$
E_{\alpha, \beta}^{\gamma, q ; c}(z)=\sum_{n=0}^{\infty} \frac{\mathbf{B}_{p}(\gamma+n q, c-\gamma)(c)_{n q}}{\mathbf{B}(\gamma, c-\gamma) \Gamma(\alpha n+\beta)} \frac{z^{n}}{n !}
$$

Corollary 3.1. Let $y, c, \rho, j, \alpha, \beta, \gamma \in \mathbb{C}$; with $\Re(\alpha)>\max \{0, \Re(c)-1\} ; \Re(c)>0 . \Re(\gamma)>$ $0, \Re(2 \rho+j)>0$ and $x>0$. Then the following formula holds true:

$$
\int_{0}^{1} x^{(\rho+j-1)}(1-x)^{(2 \rho-1)}\left(1-\frac{x}{3}\right)^{(2(\rho+j)-1)}\left(1-\frac{x}{4}\right)^{(\rho-1)} E_{\alpha, \beta}^{\gamma}\left(y\left(1-\frac{x}{4}\right)(1-x)^{2}\right) d x
$$




$$
=\left(\frac{2}{3}\right)^{2(\rho+j)} \frac{\Gamma(\rho+j)}{\Gamma(\gamma)}{ }_{2} \psi_{2}\left[\begin{array}{l}
(\rho, 1),(\gamma, 1) ; \\
(\beta, \alpha),(2 \rho+j, 1) ;
\end{array}\right.
$$

Corollary 3.2. Let $y, c, \rho, j, \alpha, \beta, \gamma \in \mathbb{C}$; with $\Re(\alpha)>\max \{0, \Re(c)-1\} ; \Re(c)>0 . \Re(\gamma)>$ $0, \Re(2 \rho+j)>0$ and $x>0$. Then the following formula holds true:

$$
\begin{gathered}
\int_{0}^{1} x^{(\rho-1)}(1-x)^{2(\rho+j)-1}\left(1-\frac{x}{3}\right)^{(2 \rho-1)}\left(1-\frac{x}{4}\right)^{(\rho+j-1)} E_{\alpha, \beta}^{\gamma}\left(y x\left(1-\frac{x}{3}\right)^{2}\right) d x \\
=\left(\frac{2}{3}\right)^{2(\rho)} \frac{\Gamma(\rho+j)}{\Gamma(\gamma)}{ }_{2} \psi_{2}\left[\begin{array}{c}
(\rho, 1),(\gamma, 1) ; \\
(\beta, \alpha),(2 \rho+j, 1) ;
\end{array}\right] .
\end{gathered}
$$

Corollary 3.3. Let $y, c, \rho, j, \alpha, \beta, \gamma \in \mathbb{C}$; with $\Re(\alpha)>\max \{0, \Re(c)-1\} ; \Re(c)>0 . \Re(\gamma)>$ $0, \Re(2 \rho+j)>0$ and $x>0$. Then the following formula holds true:

$$
\begin{gathered}
\int_{0}^{1} x^{(\rho+j-1)}(1-x)^{(2 \rho-1)}\left(1-\frac{x}{3}\right)^{(2(\rho+j)-1)}\left(1-\frac{x}{4}\right)^{(\rho-1)} E_{\alpha, \beta}^{\gamma, q}\left(y\left(1-\frac{x}{4}\right)(1-x)^{2}\right) d x \\
=\left(\frac{2}{3}\right)^{2(\rho+j)} \frac{\Gamma(\rho+j)}{\Gamma(\gamma)}{ }_{2} \psi_{2}\left[\begin{array}{c}
(\rho, 1),(\gamma, q) ; \\
(\beta, \alpha),(2 \rho+j, 1) ;
\end{array}\right]
\end{gathered}
$$

Corollary 3.4. Let $\rho, j, \alpha, \beta, \gamma \in \mathbb{C}$; with $\Re(\alpha)>0, \Re(c)>\Re(\gamma)>0, \Re(2 \rho+j)>0$ and $x>0$. Then the following formula holds true:

$$
\begin{gathered}
\int_{0}^{1} x^{(\rho-1)}(1-x)^{2(\rho+j)-1}\left(1-\frac{x}{3}\right)^{(2 \rho-1)}\left(1-\frac{x}{4}\right)^{(\rho+j-1)} E_{\alpha, \beta}^{\gamma, q}\left(y x\left(1-\frac{x}{3}\right)^{2}\right) d x \\
=\left(\frac{2}{3}\right)^{2(\rho)} \frac{\Gamma(\rho+j)}{\Gamma(\rho)}{ }_{2} \psi_{2}\left[\begin{array}{c}
(\rho, 1),(\gamma, q) ; \\
(\beta, \alpha),(2 \rho+j, 1) ;
\end{array}\right] .
\end{gathered}
$$

\section{REFERENCES}

[1] D. Baleanu, K. Diethelm, E. Scalas, J. J. Trujillo, Fractional Calculus Models and Numerical Methods, World Sci., 3 (2012), 10-16.

[2] J. Choi and P. Agarwal, Certain unified integrals associated with Bessel functions, Boundary Value Problems 2013 (2013), 95.

[3] J. Choi, P. Agarwal, S. Mathur and S.D. Purohit, Certain new integral formulas involving the generalized Bessel functions, Bull. Korean Math. Soc. 51 (2014), 995-1003.

[4] M.M. Dzrbasjan, Integral Transforms and Representations of Functions in the Complex Domain, Nauka, Moscow, (in Russian), (1966). 
[5] R. Gorenflo, F. Mainardi, Fractional calculus: integral and differential equations of fractional order, in: A. Carpinteri, F. Mainardi (Eds.), Fractals and Fractional Calculus in Continuum Mechanics, Springer Series on CSM Courses and Lectures, vol. 378, Springer-Verlag, Wien, (1997), 223276.

[6] R. Gorenflo, F. Mainardi, H.M. Srivastava, Special functions in fractional relaxationoscillation and fractional diffusion-wave phenomena, in: D. Bainov (Ed.), Proceedings of the Eighth International Colloquium on Differential Equations (Plovdiv, Bulgaria; August 1823, 1997), VSP Publishers, Utrecht and Tokyo, (1998), 195202.

[7] R. Gorenflo, A.A. Kilbas, S.V. Rogosin, On the generalized MittagLeffler type functions, Integral Transform. Spec. Funct. 7, (1998) 215224.

[8] R. Gorenflo, Y. Luchko, F. Mainardi, Wright functions as scale-invariant solutions of the diffusionwave equation, J. Comput. Appl. Math. 118 (2000), 175191.

[9] R. Hilfer, H. Seybold, Computation of the generalized MittagLeffler function and its inverse in the complex plane, Integral Transform. Spec. Funct. 17 (2006), 637652.

[10] A.A. Kilbas, M. Saigo, On MittagLeffler type function, fractional calculus operators and solutions of integral equations, Integral Transform. Spec. Funct. 4 (1996) 355370.

[11] A.A. Kilbas, H.M. Srivastava, J.J. Trujillo, Theory and Applications of Fractional Differential Equations, North-Holland Mathematical Studies, vol. 204, Elsevier (North-Holland) Science Publishers, Amsterdam, (2006).

[12] M. Saigo, A.A. Kilbas, On MittagLeffler type function and applications, Integral Transform. Spec. Funct. 7 (1998), 97112.

[13] J. L. Lavoie and G. Trottier, On the sum of certain Appells series, Ganita 20(1) (1969), 31-32.

[14] N. Menaria, S. D. Purohit and R. K. Parmar, On a new class of integrals involving generalized Mittag-Lefler function, Surveys in Mathematics and its Applications 11 (2016), 1-9.

[15] E. Mittal, R. M. Pandey, S. Joshi, On extension of Mittag-Leffler function, Appl. Appl. Math. Vol. 11, 1 (2016), pp. $307-316$.

[16] K. S. Nisar, S. D. Purohit, S. R. Mondal, Generalized fractional kinetic equations involving generalized Struve function of the first kind, J. King Saud Univ. Sci., 28 (2016), 167-171.

[17] K. S. Nisar and S. R. Mondal, Certain unified integral formulas involving the generalized modified k-Bessel function of first kind, Commun. Korean Math. Soc. 32 (2017), No. 1, pp. 4753.

[18] K. S. Nisar, P. Agarwal and S. Jain, Some unified integrals associated with Bessel-Struve kernel function, arXiv:1602.01496v1 [math.CA].

[19] M. A. Ozarslan, B. Ylmaz, The extended Mittag-Leffler function and its properties, J. Inequal. Appl., 2014:85, (2014).

[20] I. Podlubny, Fractional Differential Equations. An introduction to fractional derivatives, fractional differential equations, to methods of their solution and some of their applications, Academic Press, San Diego, CA, (1999).

[21] T.R. Prabhakar, A singular integral equation with a generalized MittagLeffler function in the kernel, Yokohama Math. J. 19 (1971), 715.

[22] S. D. Purohit, Solutions of fractional partial differential equations of quantum mechanics, Adv. Appl. Math. Mech., 5 (2013), 639-651.

[23] S. C. Sharma and Menu Devi, Certain Properties of Extended Wright Generalized Hypergeometric Function, Annals of Pure and Applied Mathematics Vol. 9, No. 1, 2015, 45-51 
[24] H.J. Seybold, R. Hilfer, Numerical results for the generalized MittagLeffler function, Fract. Calc. Appl. Anal. 8 (2005), 127139.

[25] H.M. Srivastava, A contour integral involving Foxs H-function, Indian J. Math. 14( 1972), 16.

[26] H.M. Srivastava, A note on the integral representation for the product of two generalized Rice polynomials, Collect. Math. 24 (1973), 117121.

[27] H.M. Srivastava, K.C. Gupta, S.P. Goyal, The H-Functions of One and Two Variables with Applications, South Asian Publishers, New Delhi and Madras, (1982).

[28] H.M. Srivastava, C.M. Joshi, Integral Representation For The Product Of A Class Of Generalized Hypergeometric Polynomials, Acad. Roy. Belg. Bull. Cl. Sci. (Ser. 5) 60 (1974), 919926.

[29] H.M. Srivastava, Z. Tomovski,? Fractional calculus with an integral operator containing a generalized MittagLeffler function in the kernel, Appl. Math. Comput. 211 (2009), 189-210.

[30] V. V. Uchaikin, Fractional Derivatives for Physicists and Engineers, Volume I, Background and Theory Volume II Applications, Nonlinear Physical Science, Springer-Verlag, BerlinHeidelberg, (2013).

Gauhar Rahman: Department of Mathematics, International Islamic University, IsLAMABAD, PAKISTAN

E-mail address: gauhar55uom@gmail.com

A. Ghaffar: Department of Mathematical Science, Buitems Quetta, Pakistan

E-mail address: abdulghaffar.jaffar@gmail.com

Kottakkaran Sooppy Nisar: Department of Mathematics, College of Arts and ScienceWadi Al-dawaser, 11991, Prince Sattam bin Abdulaziz University, Alkharu, Kingdom of Saudi Arabia

E-mail address: ksnisar1@gmail.com, n.sooppy@psau.edu.sa

Shahid Mubeen: Department of Mathematics, University of Sargodha, Sargodha, PakISTAN

E-mail address: smjhanda@gmail.com 